\title{
Staubmessungen am Arbeitsplatz als Basis für technische Massnahmen
}

\author{
A. Steinegger \\ Schweizerische Aluminium $A G$ \\ 8034 Zurich 4
}

Werden Messresultate der Staubkonzentration am Arbeitsplatz als Entscheidungsunterlage für techn. Verbesserungen wie Absaugung, Einkapselung etc. herangezogen, so ist es wichtig zu wissen, auf welche Weise sie zu Stande gekommen sind. Die Messergebnisse hängen van verschiedenen Faktoren ab. Die Einfluisse der Probenahmemethode und vor allem der Analytik sind eher bescheiden. Um Grössenordnungen höher jedoch sind die Faktoren, welche direkt durch den Arbeitsplatz bedingt sind.

- Arbeitsablauf, gegeben durch techn. Instailationen, vorgeschriebene Tätigkeiten, Störungen

- Einsatzstoffe z.B. verschieden stark staubendes Material

- Luftströmungen (vor allem bei stationärer Probenahme) und Luft temperatur

- individuelle Faktoren (vor allem beim Personal

Sampling) wie Arbeitsweise und Motivation.

Sind diese Einflussgrössen bekannt so ist folgende Vorgehensweise vorzusch 1 agen:

- Behebung von offensichtlichen Mänge $n$ an den techn. Einrichtungen

- Verbesserung des Unterhalts

- Motivation der Mitarbeiter

Falls diese ersten, Z.T. billigen Massnahmen nicht den erwuinschten Erfolg haben, so ist messtechnisch zu ermitteln, wie die Totalexposition zu Stande kommt. Dabei ist zu unterscheiden:

- Anteir Langzeitexposition, welcher durch die allg. Arbeitsplatzatmosphäre bestimmt wird, in der sich der Beschäftigte zum grössten Teil seiner Zeit aufhält und

- Anteil von Kurzzeitexpositionen (= einzelne Beschäftigungen) der sehr hoch sein kann z.B. bei Unterhaitsarbeiten, Behebung von Störungen etc.

In der Praxis kann sehr oft mit einer Reduktion der Staubbelastung an einzelnen Arbeitsplätzen, wo sich der Beschäftigte nur kurze zeit aufhält, sehr viel erreicht werden. Dies ist auch in Beispiel 1 gezeigt. Aus Beispiel 2 geht jedoch hervor, dass eine verbesserung des allg. Arbeitsplatzklimas in der Halle z.B. durch Luiftung und Kapselung zwar einen Erfolg bezüglich des allgemeinen Klimas zur Folge haben kann, die Exposition des Einzelnen durch die Reduktion des Langzeitanteiles aber nur unwesentlich verbessert wird.

\section{Abstract}

Measurements of dust concentration can be used as a basis for deciding on specific measures. Knowing the factors which can strongly influence the measurements is in many cases the prerequisite for finding an effective solution for eliminating too high dust exposure at reasonable cost.

$100 \%$

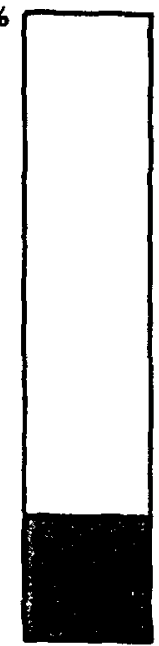

Total Exposition

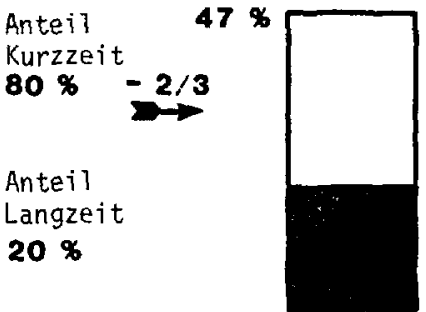

$27 \%$

Beispie1 1: Durch Reduktion des Kurzzeitanteils, urspruinglich $80 \%$ der Totalexposition, um $2 / 3$ wird die Gesamtexposition auf $47 \%$ reduziert.
$100 \%$

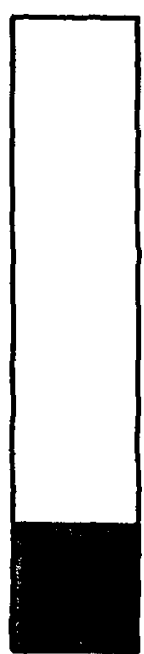

Total Exposition

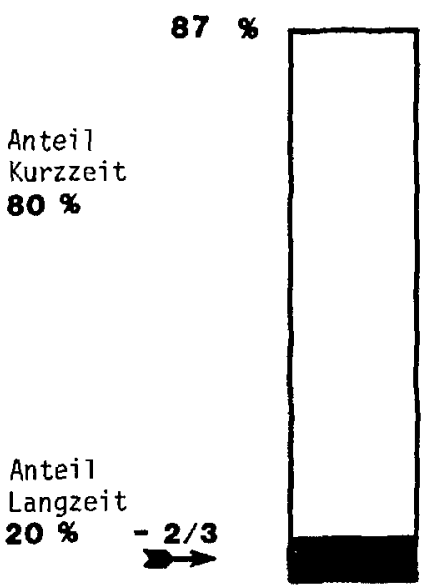

Beispiel 2: Durch Reduktion des Langzeitanteils, z.B. Hallen-Klima, um $2 / 3$ wird die Gesamtexposition nur auf $87 \%$ reduziert. 